\title{
Does the intercept of the heat-stress relation provide an accurate estimate of cardiac activation heat?
}

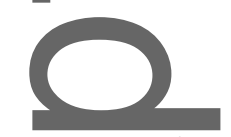

Joan Pham ${ }^{1}$, Kenneth Tran $^{2}$, Kimberley M Mellor ${ }^{1}$, Anthony Hickey ${ }^{3}$, Amelia Power ${ }^{1}$, Marie-Louise Ward ${ }^{1}$, Andrew Taberner ${ }^{2,4}$, June-Chiew Han ${ }^{2}$, Denis Loiselle ${ }^{1,2}$

Departmentoremsisio

Department of Engineering Science ${ }^{4}$, The University of Auckland

Running Title: The cost of activating contraction in cardiac muscle

Key Words: crossbridge-dependent heat, crossbridge-independent heat, length-dependence of muscle hea

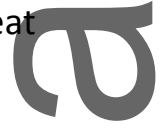

Corresponding Author: Denis Loiselle, School of Medical Sciences, Faculty of Medical and Health Sciences, The University of Auckland, Private Bag 92019, Auckland, New Zealand

Table of Contents category: Cardiovascular

Key points summary:

- The heat of activation of cardiac muscle reflects the metabolic cost of restoring ionic homeostasis following a contraction. The accuracy of its measurement depends critically on the abolition of crossbridge cycling.

- We abolished crossbridge activity in isolated rat ventricular trabeculae by use of blebbistatin, an agent that selectively inhibits myosin II ATPase.

- We find cardiac activation heat to be muscle length-independent and to account for $15-20 \%$ of total heat production at body temperature.

This is the author manuscript accepted for publication and has undergone full peer review but has not been through the copyediting, typesetting, pagination and proofreading process, which may lead to differences between this version and the Version of Record. Please cite this article as doi:

10.1113/JP274174.

This article is protected by copyright. All rights reserved. 
- We conclude that it can be accurately estimated at minimal muscle length.

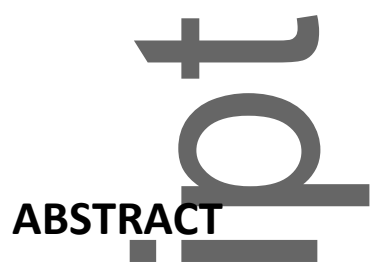

Activation heat arises from two sources during the contraction of striated muscle. It reflects the metabolic expenditure associated with $\mathrm{Ca}^{2+}$ pumping by the sarcoplasmic reticular $\mathrm{Ca}^{2+}$ ATPase and $\mathrm{Ca}^{2+}$ translocation by the $\mathrm{Na}^{+}-\mathrm{Ca}^{2+}$ exchanger coupled to the $\mathrm{Na}^{+}-\mathrm{K}^{+}$ATPase. In cardiac preparations, investigators are constrained to estimate its magnitude by reducing muscle length to the point where macroscopic twitch force vanishes. But this experimental protocol has been criticised since, at zero force, the observed heat may be contaminated by residual cross-bridge cycling activity. To eliminate this concern, the putative thermal contribution from cross-bridge cycling activity must be abolished, at least at minimal muscle length. We achieve this using blebbistatin, a selective inhibitor of myosin II ATPase. Using a microcalorimeter, we measured the force production and heat output, as functions of muscle length, of isolated rat trabeculae from both ventricles contracting isometrically at $5 \mathrm{~Hz}$ and at $37^{\circ} \mathrm{C}$. In the presence of blebbistatin $\left(15 \mu \mathrm{mol} \mathrm{L}{ }^{-1}\right)$, active force was zero but heat output remained constant, at all muscle lengths. Activation heat measured in the presence of blebbistatin was not different from that estimated from the intercept of heat-stress relation in its absence. We thus reach two conclusions. First, activation heat is independent of muscle length. Second, residual cross-bridge heat is negligible at zero active force; hence, the intercept of the cardiac heat-force relation provides an estimate of activation heat uncontaminated by crossbridge cycling. Both results resolve long-standing disputes in the literature.

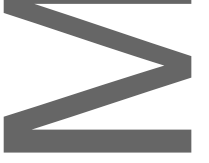

\section{INTRODUCTION}

In order for the heart to generate the next beat in a sequence, it must first be roused from its interbeat diastolic slumber. This is achieved by a 'double-triggering' sequence, the first step of which is the spread of the cardiac action potential that, via depolarisation of transverse tubular sarcolemmal membranes, mediates the inflow of $\mathrm{Ca}^{2+}$ from the extracellular space and the sarcoplasmic reticulum in a process known as $\mathrm{Ca}^{2+}$-induced $\mathrm{Ca}^{2+}$-release. It is the resulting spike in intracellular calcium concentration $\left(\left[\mathrm{Ca}^{2+}\right]_{i}\right)$ that, in turn, activates contractile proteins and contraction of the heart. In order to terminate the resulting 'active state' (Brady, 1968, Hill, 1950), thereby re-achieving diastole, $\left[\mathrm{Ca}^{2+}\right]_{i}$ must be decreased to its diastolic level and electrical equilibrium re-established. The latter is achieved by the sarcolemmal $\mathrm{Na}^{+}-\mathrm{K}^{+}$ATPase, the former by the combined efforts of the sarcolemmal $\mathrm{Ca}^{2+}$-ATPase and $\mathrm{Na}^{+}-\mathrm{Ca}^{2+}$ exchanger, and the sarcoplasmic reticular (SR) ATPase (SERCA). Each of these membrane ion transporters directly or indirectly consumes ATP. It is the summed thermal outputs of this ATP consumption, plus ATP replenishment by mitochondrial oxidative phosphorylation, that is known collectively as 'activation heat'.

The conceptual formulation of activation heat in skeletal muscle, well before its ionic basis was understood, is attributable to AV Hill $(1949,1950)$, while its formulation in cardiac muscle is credited to Chapman \& Gibbs (1972). Its method of measurement in skeletal muscle exploits the (inverse) 
linear dependence of active force on sarcomere length throughout the complete descending limb of the force-length relation (Gordon, et al., 1966). Selected skeletal muscles can be reversibly stretched beyond optimal length to the point where active force development is negligible. Thus any heat at such a muscle length may unambiguously be attributed to the 'activation' processes detailed above (Homsher, et al., 1972, Smith, 1972). The luxury of mimicking this experimental protocol does not extend to cardiac muscle since its sarcomere length can not be increased beyond approximately $2.4 \mu \mathrm{m}$ (ter Keurs, et al., 1980) without risk of irreversible damage. Hence, any 'pre-shortening' protocol must necessarily be applied on the ascending limb. Under this protocol, an isolated cardiac muscle preparation (papillary muscles (Ricchiuti \& Gibbs, 1965)) or trabeculae carneae (Han, et al., 2010, Johnston, et al., 2015), can readily be shortened to the point where no macroscopic active force appears in response to an applied stimulus. The resulting heat production, above that of basal heat, has been labelled 'activation heat'. Nevertheless, there remains unease regarding the possible contribution of actively cycling cross-bridges on any myosin filaments abutting the z-lines, in the absence of macroscopic active force development; that is, the pre-shortening procedure may consistently over-estimate the value of activation heat in cardiac muscle.

We have circumvented this risk by using blebbistatin, a selective inhibitor of myosin II ATPase (Allingham, et al., 2005). It acts by preferentially binding to the ATPase active site, while ADP and Pi are still bound, thereby greatly inhibiting phosphate release (Kovács, et al., 2004). Blebbistatin has been shown to inhibit cross-bridge cycling in slow-twitch skeletal muscle (Barclay, 2012, Lewis \& Barclay, 2014) and in murine papillary muscles and myocytes (Dou, et al., 2007), as well as in rabbit psoas myofibrils, intact and skinned rat cardiac trabeculae and myocytes (Farman, et al., 2008). Blebbistatin has noeffect on either the $\mathrm{Ca}^{2+}$-sensitivity of the contractile filaments (Dou, et al., 2007) or excitation-contraction coupling (Farman, et al., 2008).

The specific aims of our study were: (i) to measure cardiac activation heat uncontaminated by crossbridge cycling, (ii) to determine whether activation heat is ventricle-independent at body temperature and (iii) to determine whether activation heat is muscle length-dependent. We achieved these aims by use of blebbistatin.

\section{METHODS \\ Muscle preparation}

Male Wistar rats $(n=8$; age: 8-10 weeks, weight: $250-350 \mathrm{~g}$ ) were deeply anaesthetised with isoflurane, followed by cervical dislocation, thoracotomy, and cardiectomy. The heart was rapidly excised and Langendorff-perfused (at room temperature) with oxygenated Tyrode dissection solution containing (in mmol L-1): $130 \mathrm{NaCl}, 6 \mathrm{KCl}, 1 \mathrm{MgCl}_{2}, 0.5 \mathrm{NaH}_{2} \mathrm{PO}_{4}, 0.3 \mathrm{CaCl}_{2}, 10 \mathrm{HEPES}, 10$ glucose, $20 \mathrm{BDM}$ ( $\mathrm{pH}$ adjusted to 7.4 using Tris). Experiments were conducted in accordance with protocols approved by the University of Auckland Animal Ethics Committee.

Unbranched and geometrically-uniform trabeculae were dissected from the endocardial surfaces of both the left- and right- ventricles. A suitable trabecula was transferred to the mounting-chamber of a calorimeter (Han, et al., 2010, Taberner, et al., 2011). The muscle was superfused with the same oxygenated Tyrode solution but in the absence of BDM and with $\left[\mathrm{Ca}^{2+}\right]_{0}$ increased to $1.5 \mathrm{mmol} \mathrm{L}^{-1}$. 
The superfusate flow rate through the measurement chamber was electronically maintained at $0.5 \mu \mathrm{L} \mathrm{s}^{-1}$; this flow rate provides adequate oxygenation while maximising the thermal signal-tonoise ratio (Han, et al., 2011). The use of isolated cardiac trabeculae (with a typical radius less than $150 \mu \mathrm{m}$ ) obviates any concerns regarding the intrinsic complexity of the ventricle and the adequacy of oxygen supply by diffusion, even under high rates of metabolic demand (Han, et al., 2011, ter Keurs, et al., 1980)

\section{Flow-through microcalorimeter and infusion system}

Force and heat measurements were simultaneously recorded using a muscle calorimeter as previously described (Johnston, et al., 2015, Taberner, et al., 2011). The muscle was held between two platinum hooks connected to a custom-built force transducer at one end and a linear motor at the other. The Tyrode superfusate flowed over the muscle. The rate of muscle heat production was calculated from the flow-rate dependent temperature sensitivity and the difference in temperature measured between up-stream and down-stream thermopile arrays. Muscle force was monitored using a custom laser interferometer-based force transducer, as described previously (Taberner, et al., 2011). A mirror was located at $45^{\circ}$ to the axis of the measurement chamber to allow muscle diameter to be measured in two orthogonal views. All software was written in the LabVIEW programming environment (National Instruments). During experiments, the calorimeter was located inside an optically isolated and thermally insulated enclosure to ensure that experimental temperature was maintained at $37^{\circ} \mathrm{C}$.

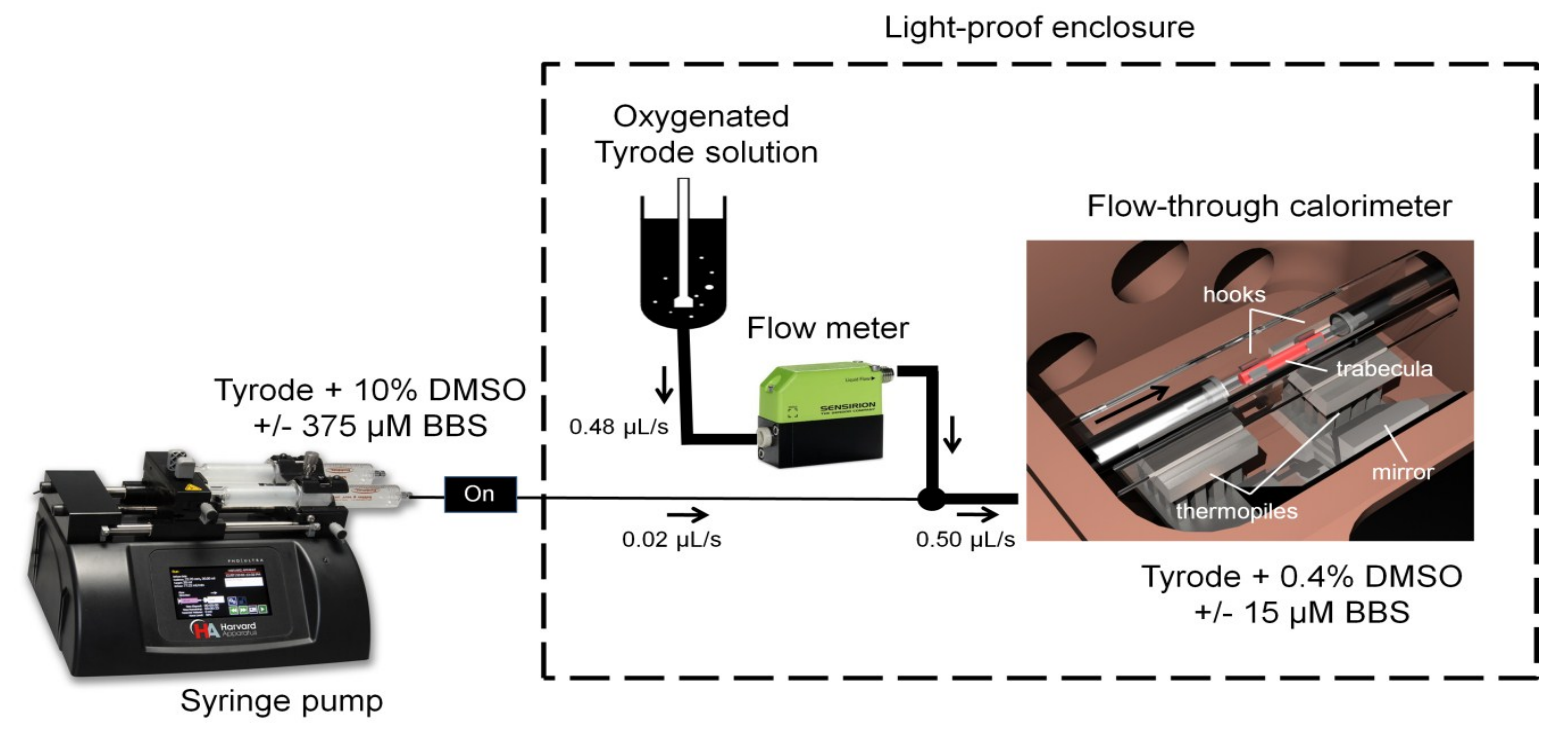

Figure 1: Schematic diagram (not to scale) of the calorimeter (located in an isolated enclosure) coupled to the infusion system (syringe pump). The arrow indicates the direction of superfusate flow. Note the 4:100 dilution of drugs in the superfusate.

A computer-controllable syringe pump was used to infuse concentrated solution containing DMSO (dimethyl sulfoxide) or blebbistatin (dissolved in DMSO) into the flowing oxygenated Tyrode solution (Figure 1). The final concentration of DMSO/blebbistatin $\left(0.4 \%\right.$ and $15 \mu \mathrm{mol} \mathrm{L}^{-1}$, respectively) in the measurement chamber of the calorimeter was readily achieved by adjusting the flow rates from the syringe pump and the oxygenated Tyrode reservoir (Taberner, et al., 2017). By ensuring that the infusate joined the superfusate well upstream of the point-of-entry to the measurement chamber (solid black symbol, Figure 1), the heat of mixing had dissipated before the mixture encountered the upstream thermopile.

This article is protected by copyright. All rights reserved. 


\section{Experimental protocols}

A trabecula was electrically stimulated to contract at $3 \mathrm{~Hz}$ at slack length until reaching steady-states of force and heat and was then gradually stretched to the length $\left(L_{o}\right)$ that maximised active force production. It was then required to undergo isometric contraction at $5 \mathrm{~Hz}$ while muscle length was progressively reduced, in 5 steps from $L_{o}$ to $L_{\min }$ (the length at which developed force was zero) using the length-control motor. At each step, steady-state force and heat were simultaneously recorded over a period of $2 \mathrm{~min}$. The muscle was then re-lengthened back to $L_{o}$ where steady-state force and heat were re-measured prior to the next intervention.

The muscle remained quiescent between interventions, thereby providing baselines of zero force and zero heat. The second and third interventions repeated the above length changes but in the presence of $0.4 \%$ DMSO or $15 \mu \mathrm{mol} \mathrm{L}^{-1}$ blebbistatin (dissolved in $0.4 \% \mathrm{DMSO}$ ), respectively. DMSO (a polar aprotic molecule) was used as the solvent for blebbistatin; therefore the second intervention was carried out to test the effect of solvent (DMSO) per se on muscle performance. At the completion of each intervention, the muscle was re-lengthened back to $L_{o}$, thereby providing a test of recovery from the length-change intervention. No force recovery was observed following treatment with blebbistatin. [Para-nitroblebbistatin (a generous gift from Professor A. MálnásiCsizmadia) was used to test for force recovery because of its reported reversibility in zebra-fish embryo in vivo (Képiró, et al., 2014); however we observed that its action too was irreversible in cardiac muscle despite lengthy periods of 'wash-out'.] The heat artifact resulting from electrical stimulation at $5 \mathrm{~Hz}$ was quantified at the end of each experiment in the absence of a muscle. The rate of muscle heat production by each trabecula was corrected retrospectively for the stimulus heat.

\section{Normalisations and definitions}

Trabeculae were assumed to have elliptical cross sections. Cross-sectional area was calculated from measurements of muscle major and minor diameters in two orthogonal planes. Trabecula dimensions did not differ significantly between LV and RV in either cross-sectional area $\left(0.036 \mathrm{~mm}^{2} \pm\right.$ $0.007 \mathrm{~mm}^{2}$ and $0.030 \mathrm{~mm}^{2} \pm+0.005 \mathrm{~mm}^{2}$, respectively) or length $(3.28 \mathrm{~mm} \pm 0.26 \mathrm{~mm}$ and $2.81 \mathrm{~mm}$ $\pm 0.36 \mathrm{~mm}$, respectively). Results were obtained from 4 trabeculae from each ventricle.

Twitch force was converted to stress by dividing by muscle cross-sectional area. Active stress was defined as the peak stress developed above the diastolic level $(\mathrm{kPa})$. Heat per twitch $\left(\mathrm{kJ} \mathrm{m}^{-3}\right)$ was calculated by dividing the steady state rate of heat production $\left(\mathrm{kW} \mathrm{m}^{-3}\right)$ by the stimulus frequency $(5 \mathrm{~Hz})$ and normalizing by muscle volume. Normalised muscle length was expressed relative to optimal muscle length $\left(L / L_{o}\right)$.

\section{Data analysis}

Data were acquired using LabView software (National Instruments, Austin, USA) and analysed offline using a custom-written MATLAB program. Active stress development and muscle heat production were regressed against relative muscle length for each muscle. The resulting individual regression lines were averaged within groups (i.e., ventricles) using the random coefficient model within PROC MIXED of the SAS software package (SAS Institute Inc., Cary, NC, USA). Analysis of covariance (ANCOVA) was applied to test the main effect of "ventricle" (i.e., 'RV 'versus 'LV') and the interaction between "ventricle" and "intervention" (i.e., 'Control', 'DMSO' and 'blebbistatin + DMSO' on the average intercepts and the average slopes. Values are expressed as means $\pm \mathrm{SE}$ and $\mathrm{P}<0.05$ was considered to be statistically significant. 


\title{
RESULTS
}

Effects of muscle length on active stress and heat. Figure 2 shows the effects of muscle length on stress production and heat output in the absence or in the presence of either $0.4 \%$ DMSO or $15 \mu \mathrm{mol} \mathrm{L} \mathrm{L}^{-1}$ blebbistatin (dissolved in $0.4 \% \mathrm{DMSO}$ ). The reduction of muscle length below $L_{o}$ resulted in a decrease of both stress production and heat output. The stress production of the same muscle decreased approximated $30 \%$ in the presence of $0.4 \%$ DMSO (in accord with observations reported by Dou et al. (2007)), thereby giving a lower heat output. Blebbistatin inhibited muscle contraction to near zero level of stress at $L_{o}$, and heat production remained constant, independent of muscle length.
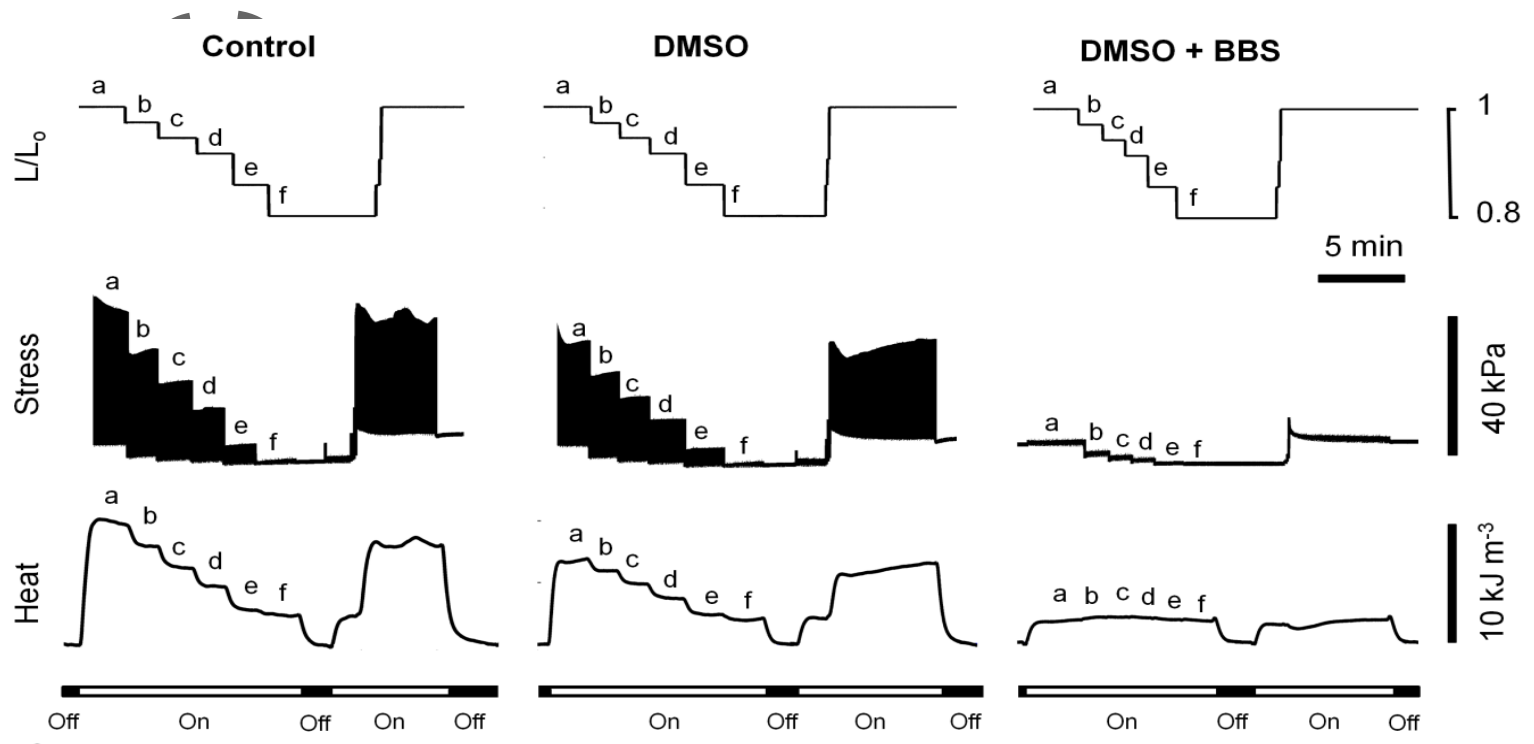

Stimulation

\begin{abstract}
Figure 2: Typical experimental records of stress production and heat output of a representative trabecula at various muscle lengths (b-f) below its optimal length (a), at $5 \mathrm{~Hz}$ stimulation. The muscle was returned to optimal length ( $\left.a^{\prime}\right)$ before performing the next two interventions: DMSO and DMSO + blebbistatin (BBS), in that order. Trabecula dimensions: length $2.82 \mathrm{~mm}$; cross-sectional area $0.0347 \mathrm{~mm}^{2}$. Its rate of heat production at $L_{o}$ under Control conditions was $4.91 \mu \mathrm{W}$. Note that the latter value exceeds the thermal resolution of the microcalorimeter by some 500 -fold.
\end{abstract}

Stress-length and heat-length relationships. As muscle length was reduced below $L_{o}$, both steadystate isometric stress and steady-state heat per twitch decreased whether in the absence or in the presence of DMSO However, heat output remained constant while active stress was absent in the presence of blebbistatin. Values of active stress and resulting heat output were plotted as functions of relative muscle length $\left(L / L_{0}\right)$ and fitted using quadratic regression (Figure $3 A \& D$ ). The relations describing the dependence of stress and heat on relative muscle length for the four trabeculae from each ventricle were averaged and are plotted in Figure 3.

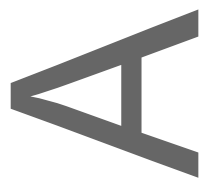

This article is protected by copyright. All rights reserved. 
A
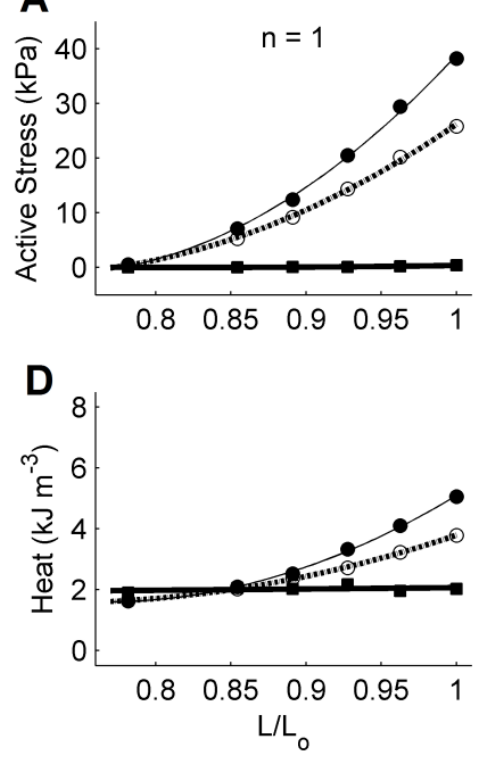

B

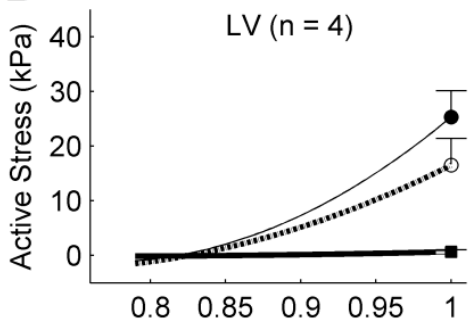

E

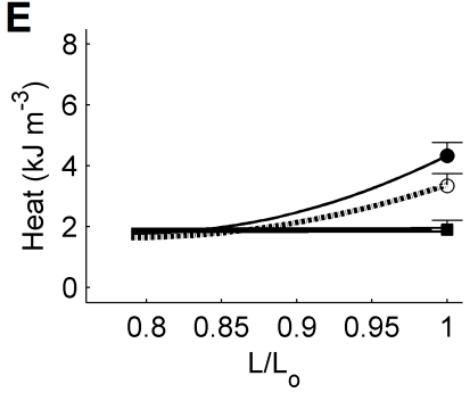

C

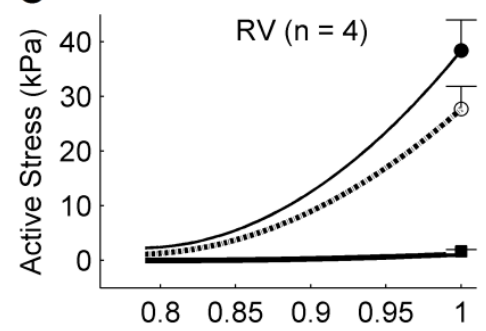

$\mathbf{F}$

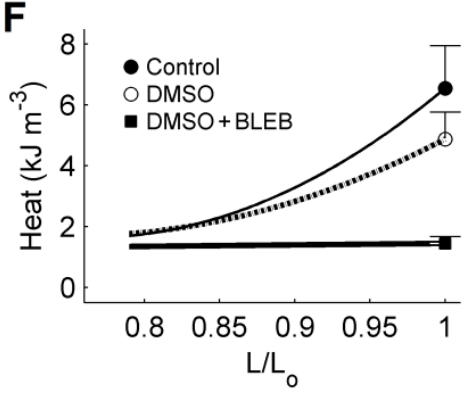

Figure 3: Active stress (A) and heat per twitch (D) as functions of muscle length from a single representative trabecula. Stress-length and heat-length regression relations describing individual muscles from the different interventions were averaged within the LV group ( $B$ and $E$ ) and the RV group ( $C$ and $F$ ). Mean \pm SEM values at optimal length were superimposed appropriately in panels B, C, E and F.

Heat-stress relationships. Figure 4 , shows the relation between active heat and active stress for a single trabecula $(A)$ and for the average of the 4 trabeculae from either ventricle ( $B$ and $C$ ). Heatstress relations were fitted using linear regression, as no statistically significant improvement was achieved when fitting with a quadratic function. The average heat-intercept (an index of activation heat) was not significantly different among experimental interventions or between LV and RV preparations (Figure 5).
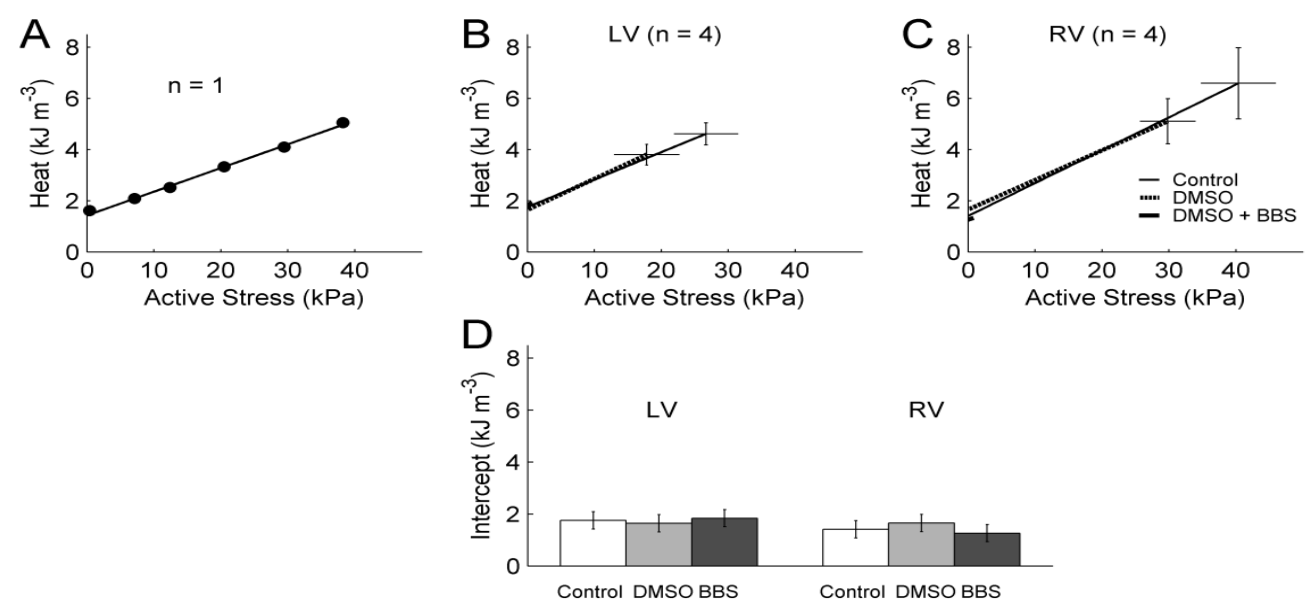

Figure 4. Twitch heat as functions of active stress. Linear regression of active heat-stress relations arising from a single trabecula under control conditions (A) and from the averages of $n=4$ trabeculae from each of LV (B) and RV (C) undergoing isometric contractions at various muscle lengths for all three experimental interventions. Panel D shows the average heat intercepts for all three treatments in trabeculae from both ventricles. 


\section{DISCUSSION}

This study is the first to have employed blebbistatin for the study of cardiac energetics. The rationale for its use was to exploit its ability to inhibit crossbridge cycling in order to partition crossbridge heat from activation heat. Activation heat is defined as the supra-basal heat produced during the restoration of sarcolemmal and sarcoplasmic reticular ion gradients associated with relaxation following a twitch. In cardiac muscle, it has commonly been assessed using the 'pre-shortening' protocol in which a preparation undergoing isometric contractions is progressively shortened until observed macroscopic force development is zero. Whatever heat is observed at this point, the intercept of the heat-stress relation, is attributed to the activation process. But this attribution immediately poses the question: "Does the absence of macroscopic force at this short muscle length belie the continued cycling of crossbridges, thereby inflating the estimate of activation heat?"

In order to answer this question, we have adopted a modification of the above protocol by repeating the pre-shortening procedure in the presence of blebbistatin (BBS), an agent shown to be an excitation-contraction uncoupler in rat and rabbit hearts (2007), and an inhibitor of crossbridge cycling in a variety of murine cardiac tissues (Dou, et al., 2007) and rat papillary muscle without diminishing the magnitude of the $\mathrm{Ca}^{2+}$ transient (Dou, et al., 2007, Farman, et al., 2008). Because of blebbistatin's insolubility in Tyrode solution, we required three interventions: 'Control' (Tyrode solution), 'DMSO' and 'DMSO+BBS' (Figure 2). As can be seen in the figure, progressive preshortening progressively diminished active twitch stress from its maximum at $L / L_{0}=1.0$ to its minimum at $L / L_{0}=0.77$ under both Control and DMSO conditions. In the presence of DMSO+BBS, active force was zero at any length, thereby proving the effectiveness of blebbistatin in inhibiting cross-bridge cycling. In the absence of active stress development, heat continued to be liberated. We attribute this thermal output (Figure 3) to 'activation heat'. Our confidence in this attribution is bolstered by the results shown in Figure 4 where the same estimates of activation heat arise from all three interventions in both LV and RV trabeculae. These results clearly demonstrate the absence of thermal contamination from crossbridge cycling.

Furthermore, our finding of constant heat output, independent of muscle length, in the presence of blebbistatin (Figures 2 and 3), demonstrates that the energy-cost of activation processes is length independent. Combining the results from Figures 3 and 4, we infer that activation heat is also stressindependent. This inference has support from the results of experiments specifically designed to increase the heat of activation of contraction. Thus Gibbs et al. (1967), using epinephrine, Gibbs and Vaughan (1968), by lowering temperature, and Han et al. (2010), by elevating extracellular $\mathrm{Ca}^{2+}$ concentration, each showed an increase of activation heat with no change in slope of the heat-stress relation.

The results summarised in Figure 4 are also fully consistent with those of Sathish et al. (2006), who showed identical peak values of $\mathrm{Ca}^{2+}$-transients elicited from myocytes harvested from right- and left-ventricular rat myocardium, albeit at room temperature. Since it is the amount of $\mathrm{Ca}^{2+}$ released from the SR that determines the amount to be sequestered by the SR $\mathrm{Ca}^{2+}$ ATPase, the correspondence of our thermal results with the aforementioned ionic results is unsurprising.

Also shown in the paper by Sathish et al. (2006) was a 50\% greater extent of unloaded shortening of myocytes originating from the LV. This result provides a possible explanation for the higher rate of

This article is protected by copyright. All rights reserved. 
'activation heat' observed (at room temperature) in LV than in RV trabeculae, reported earlier by our group (Han, et al., 2013). In those experiments, we estimated activation heat using a protocol that allowed the muscles to shorten actively under zero load. Since it has long been recognised that active shortening of striated muscle is exothermic (Fenn, 1923, Gibbs, et al., 1967, Hill, 1938), then it is again unsurprising that the heat estimated from minimally-afterloaded isotonic contractions should be higher in LV than in RV trabeculae. Since these two conceptually distinct sources of heat ('activation' and 'shortening') can not be distinguished macroscopically, we may have unwittingly over-estimated LV activation heat in our earlier publication. Be that as it may, our current findings show that activation heat accounts for some $15-20 \%$ of the total heat produced in an isometric contraction at optimal length, under conditions chosen to approximate those occurring in vivo: $5 \mathrm{~Hz}$ frequency of contraction at $37^{\circ} \mathrm{C}$.

\section{Comparison with other techniques}

In addition to studies using pre-shortening protocols, two other techniques are worthy of discussion. In the first of these, Alpert et al. (1989) measured 'tension-independent heat' in rabbit papillary muscles at $21^{\circ} \mathrm{C}$, finding it to be length-dependent. Tension-dependent heat was eliminated by use of $5 \mathrm{mM} \mathrm{2,3-butanedione} \mathrm{monoxime} \mathrm{(BDM)} \mathrm{and} \mathrm{mannitol,} \mathrm{to} \mathrm{increase} \mathrm{osmolality} \mathrm{to} \mathrm{as} \mathrm{high} \mathrm{as} \mathrm{2.5-}$ fold its normal value. We are hesitant to consider further the results of this study because subsequent investigations have demonstrated that the phosphatase BDM reduces the open probability of the L-type $\mathrm{Ca}^{2+}$ channels in guinea-pig ventricular myocytes (Allen \& Chapman, 1995), while abbreviating the duration of the action potential and reducing the $\mathrm{Ca}^{2+}$ current at any membrane potential (Gwathmey, et al., 1991). The latter study also showed that BDM reduces maximum $\mathrm{Ca}^{2+}$-activated force by upwards of $30 \%$ in ferret papillary muscles. Of further concern to any thermal study, BDM has been show to diminish the activity of the mitochondrial ATP-ADP translocase (Mojon, et al., 1993) while hyperosmolality is now known to potentiate the rate of heat production of quiescent rat cardiac trabeculae (Loiselle, et al., 1996).

A less contentious technique, 'latency release', was developed by Gibbs et al. (1988) in an attempt to obviate the risks of time-and length-dependent de-activation (Allen \& Kurihara, 1982). Using this protocol, the length of a papillary muscle was rapidly reduced from optimal to minimal muscle length during the brief latent period between delivery of the stimulus and the onset of twitch force. Since the pre-shortened length prevented the development of macroscopic force, it was presumed to quantify activation heat. But, at that time, it was not clear that the absence of macroscopic force development precludes the cycling of crossbridges.

The results of the present study strongly suggest that previous studies, in which heat-stress relations were extrapolated to zero stress, likewise produced accurate estimates of activation heat. Thus, for example, we can be confident that activation heat is lower in the rat than in either the cat or the guinea-pig [Loiselle \& Gibbs, 1979].

Limitations of the study

This article is protected by copyright. All rights reserved. 
With only 4 muscles examined from each ventricle, statistical power is low. Whereas this is correct, it is readily countered. Since all 8 trabeculae reported identical effects of blebbistatin (Figures 2-4), ethical considerations precluded the use of additional animals.

The order of presentation of treatments ('Control', 'DMSO', 'DMSO + BBS') was not randomised. This objection is again correct, but a randomised order of presentation was precluded by the inability to wash blebbistatin out of the tissues. Constraints inherent in the design of our microcalorimeter preclude the use of optical means (illumination of tissue using light in the blue region of the spectrum) to inactivate blebbistatin (Sakamoto, et al., 2005), thereby allowing reversibility of its inhibitory action on force development. Irreversibility did not, however, prevent application of a 'repeated-measures' ANOVA.

No correction was made for the change of osmolality by addition of DMSO or DMSO + blebbistatin. However, correction is unnecessary since the membrane permeability of DMSO is extremely high (some 50-fold that of urea (Yu \& Quinn, 1994)) presumably reflecting its polar nature. Whereas tonicity may have increased marginally, osmolality did not.

Finally, in the interest of completeness, we consider the possibility that, at minimal muscle length, there remains residual crossbridge heat which is below the detection threshold of our microcalorimeter. But, in fact, the thermal resolution of the microcalorimeter: $10 \mathrm{nW}$ (Taberner, et al., 2015) is three orders of magnitude below the rate of activation heat production of any trabecula use in this study.

\section{Summary}

We conclude that use of the cross-bridge inhibitor blebbistatin, despite its irreversibility in our hands, is a suitable agent for isolating a component of total cardiac heat production that can unambiguously be attributed to thermal consequence of activating contraction. Using this agent, we demonstrate that activation heat is muscle-length independent, thereby resolving a long-standing dispute in the cardiac energetics literature. But, most importantly, with the respect to the question posed in the Title, the intercept of the linear heat-stress relation provide an accurate estimate of cardiac activation heat.

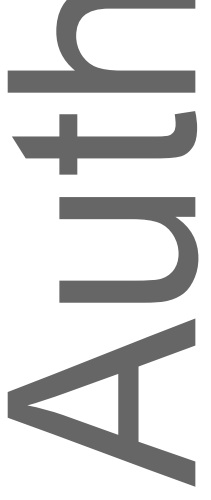

This article is protected by copyright. All rights reserved. 


\section{COMPETING INTERESTS}

We declare no competing interests.

\section{AUTHOR CONTRIBUTIONS}

Experiments were performed at the Auckland Bioengineering Institute, The University of Auckland, New Zealand.

TP: contributed to the conception and design of the work, performed the experiments, and was central to the acquisition, analysis and interpretation of data.

KT: contributed to the conception and design of the work, writing of Matlab code, analysis and interpretation of data.

KM: contributed to the conception and design of the work and interpretation of data.

$\mathrm{AH}$ : contributed to the interpretation of data.

AP: contributed to the interpretation of data.

MW: contributed to the interpretation of data.

AT: led the design, construction, programming and maintenance of the experimental apparatus, and contributed to the analysis and interpretation of data.

$\mathrm{J}-\mathrm{CH}$ : contributed to the conception and design of the work, and the acquisition, analysis and interpretation of data.

DL: contributed to the conception and design of the work, and the acquisition, analysis and interpretation of data.

All authors approved the final version of the manuscript and agree to be accountable for all aspects of the work in ensuring that questions related to the accuracy or integrity of any part of the work are appropriately investigated and resolved, and all persons designated as authors qualify for authorship, while all those who qualify for authorship are listed.

\section{FUNDING SOURCES}

This work was made possible by generous support from the National Heart Foundation of New Zealand: Project Grant 1601 (DL), Post-Doctoral Fellowships (J-CH and KT) and a PhD Scholarship (TP). DrJ-CHan is the recipient of a Fast-Start grant from the Marsden Fund, from Government funding, administered by the Royal Society of New Zealand. 


\section{REFERENCES}

Allen DG \& Kurihara S (1982). The effects of muscle length on intracellular calcium transients in mammatian cardiac muscle. J Physiol (Lond) 327, 79-94.

Allen TJA \& Chapman RA (1995). The effect of a chemical phosphatase on single calcium channels and the inactivation of whole-cell calcium current from isolated guinea-pig ventricular myocytes. Pflügers Archiv 430, 68-80.

Allingham JS, Smith R \& Rayment I (2005). The structural basis of blebbistatin inhibition and specificity for myosin II. Nat Struct Mol Biol 12, 378-379.

Alpert NR, Blanchard EM \& Mulieri LA (1989). Tension-independent heat in rabbit papillary muscle. J Physiol (Lond) 414, 433-453.

Barclay CJ (2012). Quantifying $\mathrm{Ca}^{2+}$ release and inactivation of $\mathrm{Ca}^{2+}$ release in fast- and slow-twitch muscles. J Physiol (Lond) 590, 6199-6212.

Brady AJ (1968). Active state in cardiac muscle. Physiol Rev 48, 570-600.

Chapman JB \& Gibbs CL (1972). An energetic model of muscle contraction. Biophys J 12, 227-236.

Dou Y, Arlock P \& Arner A (2007). Blebbistatin specifically inhibits actin-myosin interaction in mouse cardiac muscle. AmJ Physiol 293, C1148-C1153.

Farman GP, Tachampa K, Mateja R, Cazoria O, Lacampagne A \& de Tombe PP (2008). Blebbistatin: use as inhibitor of muscle contraction. Pflügers Archiv 455, 995-1005.

Fedorov VV, Lozinsky IT, Sosunov EA, Anyukhovsky EP, Rosen MR, Balke CW \& Efimov IR (2007). Application of blebbistatin as an excitation-contraction uncoupler for electrophysiologic study of rat and rabbit hearts. Heart Rhythm 4, 619-626.

Fenn WO (1923). A quantitative comparison between the energy liberated and the work performed by the isolated sartorius muscle of the frog. J Physiol (Lond) 58, 175-203.

Gibbs CL (1967), Role of catecholamines in heat production in the myocardium. Circ Res XX and XXI, III-223- $1 \mathrm{HH}-230$

Gibbs CL, Loiselle DS \& Wendt IR (1988). Activation heat in rabbit cardiac muscle. J Physiol (Lond) 395, 115-130.

This article is protected by copyright. All rights reserved. 
Gibbs CL, Mommaerts WFHM \& Ricchiuti NV (1967). Energetics of cardiac contractions. J Physiol (Lond) 191, 25-46.

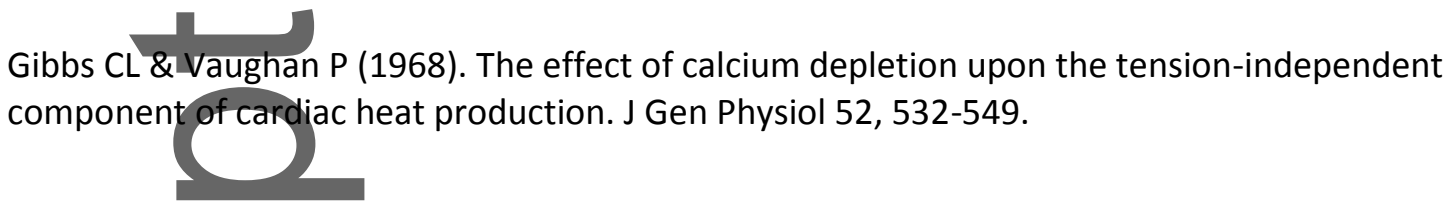

Gordon AM, Huxley AF \& Julian FJ (1966). The variation in isometric tension with sarcomere length in vertebrate muscle fibres. J Physiol (Lond) 184, 170-192.

Gwathmey JK, Hajjar RJ \& Solaro RJ (1991). Contractile deactivation and uncoupling of crossbridges. Effects of 2,3-butanedione monoxime on mammalian myocardium. Circ Res 69, 1280-1292.

Han J-C, Taberner AJ, Nielsen PMF, Kirton RS, Ward M-L \& Loiselle DS (2010). Energetics of stress production in isolated cardiac trabeculae from the rat. Am J Physiol 299, H1382-H1394.

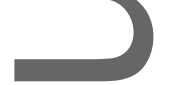

Han J-C, Taberner AJ, Nielsen PMF \& Loiselle DS (2013). Interventricular comparison of the energetics of contraction of trabeculae carneae isolated from the rat heart. Journal of Physiology (London) 591, 701-717.

Han JC, Taberner A, Kirton RS, Nielsen P, Archer R, Kim N \& Loiselle D (2011). Radius-dependent decline of performance in isolated cardiac muscle does not reflect inadequacy of diffusive oxygen supply. AmJ Physiol Heart Circ Physiol 300, H1222-H1236.

Han JC, Taberner AJ, Nielsen PM, Kirton RS, Ward ML \& Loiselle DS (2010). Energetics of stress production in isolated cardiac trabeculae from the rat. Am J Physiol Heart Circ Physiol 299, H138294.

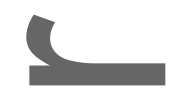

Hill AV (1938). Heat of shortening and the dynamic constants of muscle. Proceedings of the Royal Society of London B 126, 136-195.

Hill AV (1949). The heat of activation and the heat of shortening in a muscle twitch. Proceedings of the Royal Society B 136, 195-211.

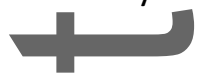

Hill AV (1950). The development of the active state of muscle during the latent period. Proceeding of the Royal Society of London Series B 137, 320-329.

Hill AV (1950). A note on the heat of activation in a muscle twitch. Proceeding of the Royal Society of London Series B 137, 330-331.

Homsher E, Mommaerts WFHM, Ricchiuti NV \& Wallner A (1972). Activation heat, activation metabolism and tension-related heat in frog semitendinosus muscles. J Physiol (Lond) 220, 601-625. 
Johnston CM, Han J-H, Ruddy BP, Nielsen PMF \& Taberner AJ (2015). A high-resolution thermoelectric-module-based calorimeter for measuring the energetics of isolated ventricular trabeculae at body temperature. Am J Physiol 309, H318-H324.

Johnston CM, Han JC, Ruddy BP, Nielsen PMF \& Taberner AJ (2015). A high-resolution thermoelectric module-based calorimeter for measuring the energetics of isolated ventricular trabeculae at body temperature. Am J Physiol Heart Circ Physiol 309, H318-H324.

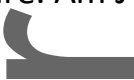

Képiró M, Vałkuti BH, Végner L, Vörös G, Hegyi G, Varga M \& Málnási-Csizmadia A (2014). paraNitroblebbistatin, the non-cytotoxic and photostable myosin II inhibitor. Angewandte Chemie 53, 16.

Kovács M, Tóth J, Hetényi C, Málnási-Csizmadia A \& Sellers JR (2004). Mechanism of Blebbistatin Inhibition of Myosin II. J Biol Chem 279, 35557-35563.

Lewis DB \& BarclayCJ (2014). Efficiency and cross-bridge work output of skeletal muscle is decreased at low levels of activation. Pflügers Archiv - European Journal of Physiology 466, 599-609.

Loiselle DS, Stienen GJM, van Hardeveld C, van der Meulen ET, Zahalak GI, Daut J \& Elzinga G (1996). The effect of hyperosmolality on the rate of heat production of quiescent trabeculae isolated from the rat heart. J Gen Physiol 108, 497-514.

Mojon D, Zhang W \& Oetliker H (1993). Inhibition by 2,3-butanedione-monoxime of mitochondrial ADP-dependent respiration and muscle contraction. Biochemistry and Molecular Biology International 31, 501-507.

Ricchiuti NV \& Gibbs CL (1965). Heat production in a cardiac contraction. Nature 208, 897-898.

Sakamoto T, Limouze J, Combs CA, Straight AF \& Sellers JR (2005). Blebbistatin, a Myosin II inhibitor, is photoinactivated by blue light. Biochemistry $44,584-588$.

Sathish $\vee, X u A$, Karmazyn M, Sims SM \& Narayanan N (2006). Mechanistic basis of differences in $\mathrm{Ca}^{2+}$-handling properties of sarcoplasmic reticulum in right and left ventricles of normal rat myocardium. American Journal of Physiology - Heart and Circulatory Physiology 291, H88-H96.

Smith ICH (1972). Energetics of activation in frog and toad muscle. J Physiol (Lond) 220, 583-599.

Taberner A, Pham T, Han JC, Uddin R \& Loiselle D (2017). A flow-through infusion calorimeter for measuring muscle energetics during pharmacological interventions. IEEE Taberner AJ, Han JC, Loiselle DS \& Nielsen PMF (2011). An innovative work-loop calorimeter for in vitro measurement of the mechanics and energetics of working cardiac trabeculae. J Appl Physiol $111,1798-1803$.

This article is protected by copyright. All rights reserved. 
ter Keurs HE, Rijnsburger WH, van Heuningen R \& Nagelsmit MJ (1980). Tension development and sarcomere length in rat cardiac trabeculae: evidence of length-dependent activation. Circulation research $46,703-714$.

ter Keurs HE, Rijnsburger WH, van Heuningen R \& Nagelsmit MJ (1980). Tension development and sarcomere length in rat cardiac traeculae. Evidence of length-dependent activation. Journal of Physiology (London) 46, 191-197.

Yu Z-W \& Quinn RJ (1994). Dimethyl sulphoxide: a review of its application in cell biology. Biosci Rep $14,259-281$

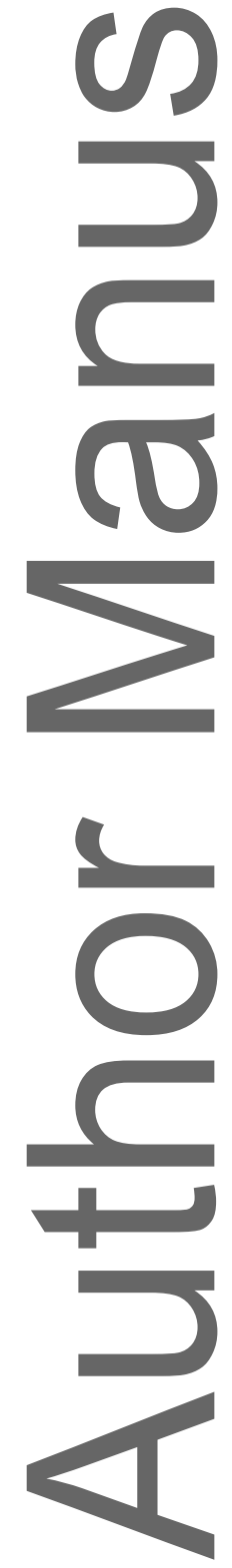




\section{University Library}

\section{- M I N E R VA}

\section{A gateway to Melbourne's research publications}

Minerva Access is the Institutional Repository of The University of Melbourne

Author/s:

Pham, T;Tran, K;Mellor, KM;Hickey, A;Power, A;Ward, M-L;Taberner, A;Han, J-C;Loiselle, D

Title:

Does the intercept of the heat-stress relation provide an accurate estimate of cardiac activation heat?

\section{Date:}

2017-07-15

\section{Citation:}

Pham, T., Tran, K., Mellor, K. M., Hickey, A., Power, A., Ward, M. -L., Taberner, A., Han, J. C. \& Loiselle, D. (2017). Does the intercept of the heat-stress relation provide an accurate estimate of cardiac activation heat?. JOURNAL OF PHYSIOLOGY-LONDON, 595 (14), pp.4725-4733. https://doi.org/10.1113/JP274174.

Persistent Link:

http://hdl.handle.net/11343/292972 